\title{
Abelian 3-form gauge theory: superfield approach
}

\author{
R. P. MALIK1 \\ Department of Physics, Centre of Advanced Studies, \\ Banaras Hindu University, Varanasi-221 005, (U. P.), India \\ and \\ DST Centre for Interdisciplinary Mathematical Sciences, \\ Banaras Hindu University, Varanasi-221 005, (U. P.), India \\ e-mails: rudra.prakash@hotmail.com, malik@bhu.ac.in
}

\begin{abstract}
We discuss a $D$-dimensional Abelian 3-form gauge theory within the framework of Bonora-Tonin's superfield formalism and derive the off-shell nilpotent and absolutely anticommuting Becchi-Rouet-Stora-Tyutin (BRST) and anti-BRST symmetry transformations for this theory. To pay our homage to Victor I. Ogievetsky (1928-1996), who was one of the inventors of Abelian 2 -form (antisymmetric tensor) gauge field, we go a step further and discuss the above $D$-dimensional Abelian 3-form gauge theory within the framework of BRST formalism and establish that the existence of the (anti-)BRST invariant Curci-Ferrari (CF) type of restrictions is the hallmark of any arbitrary $p$-form gauge theory (discussed within the framework of BRST formalism).
\end{abstract}

PACS numbers: 11.15.-q, 12.20.-m, 03.70.+k

Keywords: Bonora-Tonin's superfield formalism; Abelian 3-form gauge theory; proper (anti-)BRST symmetries; Curci-Ferrari type restrictions

\footnotetext{
${ }^{1}$ Talk delivered at BLTP, JINR, Dubna, Moscow Region, Russia in the International Workshop on "Supersymmetries and Quantum Symmetries" (SQS'11) [18-23 July 2011]. Slightly modified versions of this article were presented at International Conferences: QFT11, IISER, Pune [23-27 February 2011] and NTFT-2, BHU, Varanasi [7-12 February 2011].
} 


\section{Introduction}

In recent years, the study of higher $p$-form $(p=2,3,4, \ldots)$ gauge theories has become quite fashionable because of its relevance in the context of (super)string theories and related extended objects (see, e.g. [1,2]). It is worthwhile to mention, in this context, that Ogievetsky and Polubarinov $[3,4]$ were the first to study the 2-form antisymmetric tensor gauge field (way back in 1966-67). Our presentation is a tribute to V. I. Ogievetsky (1928-96) because we go a step further in the direction of the study of higher $p$-form $(p \geq 2)$ gauge theories and discuss the Abelian 3-form gauge theory in arbitrary $D$-dimensions of spacetime within the framework of superfield approach to Becchi-Rouet-Stora-Tyutin (BRST) formalism, proposed in $[5,6]$.

We derive the proper (i.e. off-shell nilpotent and absolutely anticommuting) (anti-)BRST symmetry transformations for the above 3 -form $\left[A^{(3)}=\right.$ $\left.(1 / 3 !)\left(d x^{\mu} \wedge d x^{\nu} \wedge d x^{\eta}\right) A_{\mu \nu \eta}\right]$ totally antisymmetric tensor gauge field $A_{\mu \nu \eta}$ which appears in the quantum excitations of the (super)strings. Furthermore, we obtain the proper (anti-)BRST transformations associated with the (anti-)ghost fields of the theory. Our main goal is to establish that the existence of the Curci-Ferrari (CF) type restrictions [7] is the hallmark of any arbitrary $p$-form $(p=1,2,3, \ldots)$ gauge theory when it is discussed within the framework of superfield approach to BRST formalism. In fact, we show that the derivation of the CF-type condition(s) is a very natural consequence of the application of the superfield approach $[5,6]$ to BRST formalism.

Our present write-up is organized as follows. In Sec. 2, we recapitulate the bare essentials of the horizontality condition and apply it to the Abelian 3-form gauge theory. In the next section, we derive the proper (anti-)BRST symmetry transformations and corresponding coupled (but equivalent) Lagrangian densities for the present theory. Our Sec. 4 deals with the critical and crucial comments on the CF type restrictions. Finally, in Sec. 5, we summarize our key results and make some concluding remarks.

\section{Horizontality Condition}

Let us begin with the starting Lagrangian density of the Abelian 3-form field

$$
\mathcal{L}_{0}=\frac{1}{24} H^{\mu \nu \eta \kappa} H_{\mu \nu \eta \kappa}, \quad H_{\mu \nu \eta \kappa}=\partial_{\mu} A_{\nu \eta \kappa}-\partial_{\nu} A_{\eta \kappa \mu}+\partial_{\eta} A_{\kappa \mu \nu}-\partial_{\kappa} A_{\mu \nu \eta}
$$

where the 4-form $H^{(4)}=d A^{(3)} \equiv(1 / 4 !)\left(d x^{\mu} \wedge d x^{\nu} \wedge d x^{\eta} \wedge d x^{\kappa}\right) H_{\mu \nu \eta \kappa}$ defines the curvature tensor $H_{\mu \nu \eta \kappa}$ which is derived from the exterior derivative $d=$ $d x^{\mu} \partial_{\mu}\left(d^{2}=0, \mu, \nu, \eta, \ldots=0,1,2, \ldots, D-1\right)$ and the 3 -form $A^{(3)}$ that encodes 
the totally antisymmetric tensor gauge connection $A_{\mu \nu \eta}$. It can be easily seen that, under the following local gauge symmetry transformations $\delta_{g}$ (with the local infinitesimal antisymmetric gauge parameter $\Lambda_{\mu \nu}=-\Lambda_{\nu \mu}$ )

$$
\delta_{g} A_{\mu \nu \eta}=\partial_{\mu} \Lambda_{\nu \eta}+\partial_{\nu} \Lambda_{\eta \mu}+\partial_{\eta} \Lambda_{\mu \nu}
$$

the Lagrangian density and the curvature tensor $H_{\mu \nu \eta \kappa}$ remain invariant. Thus, the curvature tensor $H_{\mu \nu \eta \kappa}$, derived from the 4-form $H^{(4)}$, is a physical quantity (in some sense). One of the most intuitive approaches to quantize the $p$-form gauge theory is the BRST formalism where the local gauge symmetry transformations (e.g. (2)) are traded with the proper (i.e. nilpotent and absolutely anticommuting) (anti-)BRST symmetry transformations.

The above (anti-)BRST symmetries can be derived by exploiting the geometrical superfield formalism $[5,6]$ where any arbitrary $D$-dimensional gauge theory is generalized onto the $(D, 2)$-dimensional supermanifold as [8]:

$$
\begin{aligned}
& d \longrightarrow \tilde{d} \equiv d Z^{M} \partial_{M}=d x^{\mu} \partial_{\mu}+d \theta \partial_{\theta}+d \bar{\theta} \partial_{\bar{\theta}}, \quad x^{\mu} \longrightarrow Z^{M}=\left(x^{\mu}, \theta, \bar{\theta}\right), \\
& A^{(3)} \longrightarrow \tilde{A}^{(3)}=\frac{\left(d Z^{M} \wedge d Z^{N} \wedge d Z^{K}\right)}{3 !} \tilde{A}_{M N K}, \quad \partial_{M}=\left(\partial_{\mu}, \partial_{\theta}, \partial_{\bar{\theta}}\right) .
\end{aligned}
$$

Here the superspace variables $Z^{M}=\left(x^{\mu}, \theta, \bar{\theta}\right)$ are the generalization of the ordinary $D$-dimensional coordinates $x^{\mu}$ that incorporate a pair of Grassmannian variables $\theta$ and $\bar{\theta}$, too, which satisfy $\theta^{2}=\bar{\theta}^{2}=0, \theta \bar{\theta}+\bar{\theta} \theta=0$. In the horizontality condition (HC), we require the physical (geometrical) quantity $H^{(4)}$ to remain independent of the Grassmannian variables, namely;

$$
\tilde{H}^{(4)}=H^{(4)} \Longrightarrow \tilde{d} \tilde{A}^{(3)}=d A^{(3)} .
$$

This leads, automatically, to the derivation of the off-shell nilpotent $\left(s_{(a) b}^{2}=\right.$ $0)$ and absolutely anticommuting $\left(s_{b} s_{a b}+s_{a b} s_{b}=0\right)$ (anti-)BRST symmetry transformations $\left(s_{(a) b}\right)$ for the gauge field and corresponding (anti-)ghost fields of the theory which we discuss, in detail, in the following section.

\section{Proper (Anti-)BRST Symmetries}

It is clear from equation (4) that the r.h.s. of it contains only the spacetime differentials [i.e. $d A^{(3)}=(1 / 4 !)\left(d x^{\mu} \wedge d x^{\nu} \wedge d x^{\eta} \wedge d x^{\kappa}\right) H_{\mu \nu \eta \kappa}$ ]. However, the l.h.s. contains the spacetime differentials together with the Grassmann differentials. Thus, it is evident that the $\mathrm{HC}$ amounts to setting equal to zero the coefficient of all the differentials that contain Grassmannian variables. To 
check the above statement, it is imperative to compute the l.h.s. explicitly. Towards this goal, it can be seen that the equation (3) implies [8]

$$
\begin{aligned}
& \tilde{A}^{(3)}=\frac{1}{3 !}\left(d x^{\mu} \wedge d x^{\nu} \wedge d x^{\eta}\right) \tilde{A}_{\mu \nu \eta}+\frac{1}{2}\left(d x^{\mu} \wedge d x^{\nu} \wedge d \theta\right) \tilde{A}_{\mu \nu \theta} \\
& +\frac{1}{2}\left(d x^{\mu} \wedge d x^{\nu} \wedge d \bar{\theta}\right) \tilde{A}_{\mu \nu \bar{\theta}}+\frac{1}{3 !}(d \theta \wedge d \theta \wedge d \theta) \tilde{A}_{\theta \theta \theta}+\frac{1}{3 !}(d \bar{\theta} \wedge d \bar{\theta} \wedge d \bar{\theta}) \tilde{A}_{\bar{\theta} \bar{\theta} \bar{\theta}} \\
& +\left(d x^{\mu} \wedge d \theta \wedge d \bar{\theta}\right) \tilde{A}_{\mu \theta \bar{\theta}}+\frac{1}{2}\left(d x^{\mu} \wedge d \theta \wedge d \theta\right) \tilde{A}_{\mu \theta \theta}+\frac{1}{2}(d \theta \wedge d \theta \wedge d \bar{\theta}) \tilde{A}_{\theta \theta \bar{\theta}} \\
& +\frac{1}{2}\left(d x^{\mu} \wedge d \bar{\theta} \wedge d \bar{\theta}\right) \tilde{A}_{\mu \bar{\theta} \bar{\theta}}+\frac{1}{2}(d \theta \wedge d \bar{\theta} \wedge d \bar{\theta}) \tilde{A}_{\theta \bar{\theta} \bar{\theta}}
\end{aligned}
$$

Keeping in mind the nature of the superfields, we make the suitable identifications: $\tilde{A}_{\mu \nu \eta}=\tilde{\mathcal{A}}_{\mu \nu \eta}(x, \theta, \bar{\theta}), \tilde{A}_{\mu \nu \theta}=\tilde{\mathcal{\mathcal { F }}}_{\mu \nu}(x, \theta, \bar{\theta}), \tilde{A}_{\mu \nu \bar{\theta}}=\tilde{\mathcal{F}}_{\mu \nu}(x, \theta, \bar{\theta}), \tilde{A}_{\mu \theta \bar{\theta}}=$ $\tilde{\Phi}_{\mu}(x, \theta, \bar{\theta}), \frac{1}{3 !} \tilde{A}_{\theta \theta \theta}=\tilde{\mathcal{F}}_{2}(x, \theta, \bar{\theta}), \frac{1}{3 !} A_{\bar{\theta} \bar{\theta} \bar{\theta}}=\tilde{\mathcal{F}}_{2}(x, \theta, \bar{\theta}), \frac{1}{2} \tilde{A}_{\theta \bar{\theta} \bar{\theta}}=\tilde{\mathcal{F}}_{1}(x, \theta, \bar{\theta})$, $\frac{1}{2} \tilde{A}_{\theta \theta \bar{\theta}}=\tilde{\mathcal{F}}_{1}(x, \theta, \bar{\theta}), \frac{1}{2} \tilde{A}_{\mu \bar{\theta} \bar{\theta}}=\tilde{\beta}_{\mu}(x, \theta, \bar{\theta})$ and $\frac{1}{2} \tilde{A}_{\mu \theta \theta}=\tilde{\bar{\beta}}_{\mu}(x, \theta, \bar{\theta})$ as the generalization of the $D$-dimensional local fields $A_{\mu \nu \eta}, \bar{C}_{\mu \nu}, C_{\mu \nu}, \phi_{\mu}, \bar{C}_{2}, C_{2}, C_{1}, \bar{C}_{1}, \beta_{\mu}$, $\bar{\beta}_{\mu}$ of the (anti-)BRST invariant local $D$-dimensional ordinary theory onto the $(D, 2)$-dimensional supermanifold (within our superfield formalism).

The super-expansions of the above superfields, along the Grassmannian directions of the $(D, 2)$-dimensional supermanifold are as follows [8]:

$$
\begin{aligned}
\tilde{\mathcal{A}}_{\mu \nu \eta}(x, \theta, \bar{\theta}) & =A_{\mu \nu \eta}(x)+\theta \bar{R}_{\mu \nu \eta}(x)+\bar{\theta} R_{\mu \nu \eta}(x)+i \theta \bar{\theta} S_{\mu \nu \eta}(x), \\
\tilde{\beta}_{\mu}(x, \theta, \bar{\theta}) & =\beta_{\mu}(x)+\theta \bar{f}_{\mu}^{(1)}(x)+\bar{\theta} f_{\mu}^{(1)}(x)+i \theta \bar{\theta} b_{\mu}(x), \\
\tilde{\bar{\beta}}_{\mu}(x, \theta, \bar{\theta}) & =\bar{\beta}_{\mu}(x)+\theta \bar{f}_{\mu}^{(2)}(x)+\bar{\theta} f_{\mu}^{(2)}(x)+i \theta \bar{\theta} \bar{b}_{\mu}(x), \\
\tilde{\Phi}_{\mu}(x, \theta, \bar{\theta}) & =\phi_{\mu}(x)+\theta \bar{f}_{\mu}^{(3)}(x)+\bar{\theta} f_{\mu}^{(3)}(x)+i \theta \bar{\theta} b_{\mu}^{(3)}(x), \\
\tilde{\mathcal{F}}_{\mu \nu}(x, \theta, \bar{\theta}) & =C_{\mu \nu}(x)+\theta \bar{B}_{\mu \nu}^{(1)}(x)+\bar{\theta} B_{\mu \nu}^{(1)}(x)+i \theta \bar{\theta} s_{\mu \nu}(x), \\
\tilde{\overline{\mathcal{F}}}_{\mu \nu}(x, \theta, \bar{\theta}) & =\bar{C}_{\mu \nu}(x)+\theta \bar{B}_{\mu \nu}^{(2)}(x)+\bar{\theta} B_{\mu \nu}^{(2)}(x)+i \theta \bar{\theta} \bar{s}_{\mu \nu}(x), \\
\tilde{\mathcal{F}}_{1}(x, \theta, \bar{\theta}) & =C_{1}(x)+\theta \bar{b}_{1}^{(1)}(x)+\bar{\theta} b_{1}^{(1)}(x)+i \theta \bar{\theta} s_{1}(x), \\
\tilde{\overline{\mathcal{F}}}_{1}(x, \theta, \bar{\theta}) & =\bar{C}_{1}(x)+\theta \bar{b}_{1}^{(2)}(x)+\bar{\theta} b_{1}^{(2)}(x)+i \theta \bar{\theta} \bar{s}_{1}(x), \\
\tilde{\mathcal{F}}_{2}(x, \theta, \bar{\theta}) & =C_{2}(x)+\theta \bar{b}_{2}^{(1)}(x)+\bar{\theta} b_{2}^{(1)}(x)+i \theta \bar{\theta} s_{2}(x), \\
\tilde{\overline{\mathcal{F}}}_{2}(x, \theta, \bar{\theta}) & =\bar{C}_{2}(x)+\theta \bar{b}_{2}^{(2)}(x)+\bar{\theta} b_{2}^{(2)}(x)+i \theta \bar{\theta} \bar{s}_{2}(x),
\end{aligned}
$$

where $A_{\mu \nu \eta}$ is the gauge field, $\phi_{\mu}$ is the vector bosonic field, $\left(\bar{C}_{\mu \nu}\right) C_{\mu \nu}$ are the pair of fermionic antisymmetric (anti-)ghost fields, $\left(\bar{\beta}_{\mu}\right) \beta_{\mu}$ are the bosonic ghost-for-ghost (anti-)ghost fields, $\left(\bar{C}_{2}\right) C_{2}$ and $\left(\bar{C}_{1}\right) C_{1}$ are the Lorentz scalar fermionic (anti-)ghost fields. The above fields are required for the proof of unitarity in the theory. The rest of the fields, on the r.h.s. of equation (6), 
are secondary fields that have to be determined in terms of the basic and auxiliary fields of the $D$-dimensional ordinary theory by exploiting the $\mathrm{HC}$.

Explicit computation of (4) and setting equal to zero all the coefficients of the Grassmannian differentials of the super 4-form of the l.h.s., leads to

$$
\begin{aligned}
& b_{2}^{(1)}=0, \quad s_{2}=0, \quad \bar{b}_{2}^{(2)}=0, \quad \bar{s}_{2}=0, \quad \bar{s}_{1}=0, \quad s_{1}=0 \\
& \bar{b}_{2}^{(1)}+b_{1}^{(1)}=0, \quad b_{1}^{(2)}+\bar{b}_{1}^{(1)}=0, \quad \bar{f}_{\mu}^{(2)}=\partial_{\mu} \bar{C}_{2}, \quad f_{\mu}^{(1)}=\partial_{\mu} C_{2}, \\
& \bar{b}_{\mu}=-i \partial_{\mu} b_{2}^{(2)}, \quad b_{\mu}^{(3)}=-i \partial_{\mu} b_{1}^{(2)}, \quad B_{\mu \nu}^{(1)}=\partial_{\mu} \beta_{\nu}-\partial_{\nu} \beta_{\mu}, \quad \bar{f}_{\mu}^{(2)}=\partial_{\mu} \bar{C}_{2}, \\
& \bar{B}_{\mu \nu}^{(2)}=\partial_{\mu} \bar{\beta}_{\nu}-\partial_{\nu} \bar{\beta}_{\mu}, \quad s_{\mu \nu}=i\left(\partial_{\mu} \bar{f}_{\nu}^{(1)}-\partial_{\nu} \bar{f}_{\mu}^{(1)}\right) \equiv-i\left(\partial_{\mu} f_{\nu}^{(3)}-\partial_{\nu} f_{\mu}^{(3)}\right), \\
& \bar{s}_{\mu \nu}=+i\left(\partial_{\mu} \bar{f}_{\nu}^{(3)}-\partial_{\nu} \bar{f}_{\mu}^{(3)}\right) \equiv-i\left(\partial_{\mu} f_{\nu}^{(2)}-\partial_{\nu} f_{\mu}^{(2)}\right), \quad b_{\mu}=i \partial_{\mu} \bar{b}_{2}^{(1)}, \\
& R_{\mu \nu \eta}=\partial_{\mu} C_{\nu \eta}+\partial_{\nu} C_{\eta \mu}+\partial_{\eta} C_{\mu \nu}, \quad \bar{R}_{\mu \nu \eta}=\partial_{\mu} \bar{C}_{\nu \eta}+\partial_{\nu} \bar{C}_{\eta \mu}+\partial_{\eta} \bar{C}_{\mu \nu}, \\
& S_{\mu \nu \eta}=-i\left(\partial_{\mu} B_{\nu \eta}^{(2)}+\partial_{\nu} B_{\eta \mu}^{(2)}+\partial_{\eta} B_{\mu \nu}^{(2)}\right) \\
& \equiv+i\left(\partial_{\mu} \bar{B}_{\nu \eta}^{(1)}+\partial_{\nu} \bar{B}_{\eta \mu}^{(1)}+\partial_{\eta} \bar{B}_{\mu \nu}^{(1)}\right), \quad b_{2}^{(2)}+\bar{b}_{1}^{(2)}=0 .
\end{aligned}
$$

In addition to the above results, we obtain the following Curci-Ferrari type restrictions from the $\mathrm{HC}$ illustrated in (4), namely;

$$
f_{\mu}^{(2)}+\bar{f}_{\mu}^{(3)}=\partial_{\mu} \bar{C}_{1}, \quad \bar{f}_{\mu}^{(1)}+f_{\mu}^{(3)}=\partial_{\mu} C_{1}, \quad \bar{B}_{\mu \nu}^{(1)}+B_{\mu \nu}^{(2)}=\partial_{\mu} \phi_{\nu}-\partial_{\nu} \phi_{\mu},
$$

which ensure the consistency of the three equivalences shown in (7). At this stage, a couple of remarks are in order. First, the above restrictions emerge from setting the specific coefficients of the 4-form differentials [e.g. $\left.\left(d x^{\mu} \wedge d \theta \wedge d \theta \wedge d \bar{\theta}\right),\left(d x^{\mu} \wedge d \theta \wedge d \bar{\theta} \wedge d \bar{\theta}\right),\left(d x^{\mu} \wedge d x^{\nu} \wedge d \theta \wedge d \bar{\theta}\right)\right]$ of the l.h.s. of the HC. Second, it is worth pointing out that the coefficients of the differentials $\left(d x^{\mu} \wedge d x^{\nu} \wedge d x^{\eta} \wedge d x^{\xi}\right)$ from the l.h.s. and r.h.s. of the condition $\tilde{d} \tilde{A}^{(3)}=d A^{(3)}$ match due to the precise form of $R_{\mu \nu \eta}, \bar{R}_{\mu \nu \eta}$ and $S_{\mu \nu \eta}$, quoted in (7).

To make the notations cute and a bit simpler, we identify: $b_{1}^{(2)}=B_{1}, b_{2}^{(2)}=$ $B_{2}, \bar{b}_{2}^{(1)}=\bar{B}, \bar{f}_{\mu}^{(1)}=F_{\mu}, f_{\mu}^{(2)}=\bar{F}_{\mu}, f_{\mu}^{(3)}=f_{\mu}, \bar{f}_{\mu}^{(3)}=\bar{f}_{\mu}, B_{\mu \nu}^{(2)}=B_{\mu \nu}, \bar{B}_{\mu \nu}^{(1)}=$ $\bar{B}_{\mu \nu}$. As a consequence, the celebrated CF type restrictions become $B_{\mu \nu}+$ $\bar{B}_{\mu \nu}=\partial_{\mu} \phi_{\nu}-\partial_{\nu} \phi_{\mu}, f_{\mu}+F_{\mu}=\partial_{\mu} C_{1}, \bar{f}_{\mu}+\bar{F}_{\mu}=\partial_{\mu} \bar{C}_{1}$. Furhermore, the proper off-shell nilpotent and absolutely anticommuting (anti-)BRST symmetry transformations that emerge from $\mathrm{HC}$ [cf. equation (4)] are [8,9]

$$
\begin{aligned}
& s_{a b} A_{\mu \nu \eta}=\partial_{\mu} \bar{C}_{\nu \eta}+\partial_{\nu} \bar{C}_{\eta \mu}+\partial_{\eta} \bar{C}_{\mu \nu}, \quad s_{a b} \bar{C}_{\mu \nu}=\partial_{\mu} \bar{\beta}_{\nu}-\partial_{\nu} \bar{\beta}_{\mu}, \\
& s_{a b} \bar{\beta}_{\mu}=\partial_{\mu} \bar{C}_{2}, \quad s_{a b} \bar{C}_{2}=0, \quad s_{a b} \bar{B}_{\mu \nu}=0, \quad s_{a b} C_{1}=-B_{1}, \\
& s_{a b} \bar{C}_{1}=-B_{2}, \quad s_{a b} \bar{B}=0, \quad s_{a b} C_{2}=\bar{B}, \quad s_{a b} \beta_{\mu}=\bar{F}_{\mu}, \quad s_{a b} \bar{F}_{\mu}=0, \\
& s_{a b} \bar{f}_{\mu}=0, \quad s_{a b} F_{\mu}=-\partial_{\mu} B_{2}, \quad s_{a b} f_{\mu}=-\partial_{\mu} B_{1}, \quad s_{a b} B_{\mu \nu}=\partial_{\mu} \bar{f}_{\nu}-\partial_{\nu} \bar{f}_{\mu}, \\
& s_{a b} C_{\mu \nu}=\bar{B}_{\mu \nu}, \quad s_{a b} B_{1}=0, \quad s_{a b} B_{2}=0,
\end{aligned}
$$




$$
\begin{aligned}
& s_{b} A_{\mu \nu \eta}=\partial_{\mu} C_{\nu \eta}+\partial_{\nu} C_{\eta \mu}+\partial_{\eta} C_{\mu \nu}, \quad s_{b} C_{\mu \nu}=\partial_{\mu} \beta_{\nu}-\partial_{\nu} \beta_{\mu}, \\
& s_{b} \beta_{\mu}=\partial_{\mu} C_{2}, \quad s_{b} C_{2}=0, \quad s_{b} B_{\mu \nu}=0, \quad s_{b} C_{1}=-\bar{B}, \\
& s_{b} \bar{C}_{1}=B_{1}, \quad s_{b} B_{1}=0, \quad s_{b} \bar{C}_{2}=B_{2}, \quad s_{b} \bar{\beta}_{\mu}=F_{\mu}, \quad s_{b} F_{\mu}=0 \\
& s_{b} f_{\mu}=0, \quad s_{b} \bar{F}_{\mu}=-\partial_{\mu} \bar{B}, \quad s_{b} \bar{f}_{\mu}=\partial_{\mu} B_{1}, \quad s_{b} \bar{B}_{\mu \nu}=\partial_{\mu} f_{\nu}-\partial_{\nu} f_{\mu}, \\
& s_{b} \bar{C}_{\mu \nu}=B_{\mu \nu}, \quad s_{b} \bar{B}=0, \quad s_{b} B_{2}=0, \quad s_{b} \phi_{\mu}=f_{\mu} .
\end{aligned}
$$

It is elementary to check that the above (anti-)BRST symmetry transformations are off-shell nilpotent of order two (i.e. $\left.s_{(a) b}^{2}=0\right)$.

The above transformations have been obtained from the superfield formalism without any knowledge of the (anti-)BRST invariant Lagrangian density. This is due to the fact that the substitution of the results of (7) into (6) leads to the following super-expansion of the superfields in the language of the nilpotent (anti-)BRST symmetry transformations [8]

$$
\begin{aligned}
\tilde{\mathcal{A}}_{\mu \nu \eta}^{(h)}(x, \theta, \bar{\theta}) & =A_{\mu \nu \eta}(x)+\theta\left(s_{a b} A_{\mu \nu \eta}(x)\right)+\bar{\theta}\left(s_{b} A_{\mu \nu \eta}(x)\right)+\theta \bar{\theta}\left(s_{b} s_{a b} A_{\mu \nu \eta}(x)\right), \\
\tilde{\beta}_{\mu}^{(h)}(x, \theta, \bar{\theta}) & =\beta_{\mu}(x)+\theta\left(s_{a b} \beta_{\mu}(x)\right)+\bar{\theta}\left(s_{b} \beta_{\mu}(x)\right)+\theta \bar{\theta}\left(s_{b} s_{a b} \beta_{\mu}(x)\right), \\
\tilde{\bar{\beta}}_{\mu}^{(h)}(x, \theta, \bar{\theta}) & =\bar{\beta}_{\mu}(x)+\theta\left(s_{a b} \bar{\beta}_{\mu}(x)\right)+\bar{\theta}\left(s_{b} \bar{\beta}_{\mu}(x)\right)+\theta \bar{\theta}\left(s_{b} s_{a b} \bar{\beta}_{\mu}(x)\right), \\
\tilde{\Phi}_{\mu}^{(h)}(x, \theta, \bar{\theta}) & =\phi_{\mu}(x)+\theta\left(s_{a b} \phi_{\mu}(x)\right)+\bar{\theta}\left(s_{b} \phi_{\mu}(x)\right)+\theta \bar{\theta}\left(s_{b} s_{a b} \phi_{\mu}(x)\right), \\
\tilde{\mathcal{F}}_{\mu \nu}^{(h)}(x, \theta, \bar{\theta}) & =C_{\mu \nu}(x)+\theta\left(s_{a b} C_{\mu \nu}(x)\right)+\bar{\theta}\left(s_{b} C_{\mu \nu}(x)\right)+\theta \bar{\theta}\left(s_{b} s_{a b} C_{\mu \nu}(x)\right), \\
\tilde{\mathcal{F}}_{\mu \nu}^{(h)}(x, \theta, \bar{\theta}) & =\bar{C}_{\mu \nu}(x)+\theta\left(s_{a b} \bar{C}_{\mu \nu}(x)\right)+\bar{\theta}\left(s_{b} \bar{C}_{\mu \nu}(x)\right)+\theta \bar{\theta}\left(s_{b} s_{a b} \bar{C}_{\mu \nu}(x)\right), \\
\tilde{\mathcal{F}}_{1}^{(h)}(x, \theta, \bar{\theta}) & =C_{1}(x)+\theta\left(s_{a b} C_{1}(x)\right)+\bar{\theta}\left(s_{b} C_{1}(x)\right)+\theta \bar{\theta}\left(s_{b} s_{a b} C_{1}(x)\right), \\
\tilde{\mathcal{F}}_{1}^{(h)}(x, \theta, \bar{\theta}) & =\bar{C}_{1}(x)+\theta\left(s_{a b} \bar{C}_{1}(x)\right)+\bar{\theta}\left(s_{b} \bar{C}_{1}(x)\right)+\theta \bar{\theta}\left(s_{b} s_{a b} \bar{C}_{1}(x)\right), \\
\tilde{\mathcal{F}}_{2}^{(h)}(x, \theta, \bar{\theta}) & =C_{2}(x)+\theta\left(s_{a b} C_{2}(x)\right)+\bar{\theta}\left(s_{b} C_{2}(x)\right)+\theta \bar{\theta}\left(s_{b} s_{a b} C_{2}(x)\right), \\
\tilde{\mathcal{F}}_{2}^{(h)}(x, \theta, \bar{\theta}) & =\bar{C}_{2}(x)+\theta\left(s_{a b} \bar{C}_{2}(x)\right)+\bar{\theta}\left(s_{b} \bar{C}_{2}(x)\right)+\theta \bar{\theta}\left(s_{b} s_{a b} \bar{C}_{2}(x)\right), \quad(11)
\end{aligned}
$$

where the proper (anti-)BRST symmetry transformations are denoted by $s_{(a) b}$ and the superscript $(h)$, on the superfields, stands for the super-expansions of these superfields obtained after the application of HC (cf. (4)).

Furthermore, it can be checked that the anticommutativity property (i.e. $\left.s_{b} s_{a b}+s_{a b} s_{b}=0\right)$ of $s_{(a) b}$ on the following basic fields [8,9]

$$
\left\{s_{b}, s_{a b}\right\} A_{\mu \nu \eta}=0, \quad\left\{s_{b}, s_{a b}\right\} C_{\mu \nu}=0, \quad\left\{s_{b}, s_{a b}\right\} \bar{C}_{\mu \nu}=0,
$$

is true only when the Curci-Ferrari type restrictions (8) are satisfied. The property of the anticommutativity of the (anti-)BRST symmetry transformations is trivially obeyed in the case of the rest of the fields of our present $D$-dimensional Abelian 3-form gauge theory. Finally, one can write down the 
coupled [but equivalent] (anti-)BRST invariant Lagrangian densities for the above Abelian 3-form gauge theory as (see, e.g. [9] for details)

$$
\begin{aligned}
\mathcal{L}_{B} & =\frac{1}{24} H^{\mu \nu \eta \xi} H_{\mu \nu \eta \xi}+s_{b} s_{a b}\left(\frac{1}{2} \bar{C}_{2} C_{2}-\frac{1}{2} \bar{C}_{1} C_{1}+\frac{1}{2} \bar{C}_{\mu \nu} C^{\mu \nu}\right. \\
& \left.-\bar{\beta}^{\mu} \beta_{\mu}-\frac{1}{2} \phi^{\mu} \phi_{\mu}-\frac{1}{6} B^{\mu \nu \eta} B_{\mu \nu \eta}\right), \\
\mathcal{L}_{\bar{B}} & =\frac{1}{24} H^{\mu \nu \eta \xi} H_{\mu \nu \eta \xi}-s_{a b} s_{b}\left(\frac{1}{2} \bar{C}_{2} C_{2}-\frac{1}{2} \bar{C}_{1} C_{1}+\frac{1}{2} \bar{C}_{\mu \nu} C^{\mu \nu}\right. \\
& \left.-\bar{\beta}^{\mu} \beta_{\mu}-\frac{1}{2} \phi^{\mu} \phi_{\mu}-\frac{1}{6} B^{\mu \nu \eta} B_{\mu \nu \eta}\right) .
\end{aligned}
$$

The first Lagrangian density $\mathcal{L}_{B}$ is trivially invariant under the BRST transformations $s_{b}$. On the other hand, the second Lagrangian density $\mathcal{L}_{\bar{B}}$ is trivially invariant under the anti-BRST symmetry transformations $s_{a b}$. One can check that, under $s_{a b}$, the first Lagrangian density $\mathcal{L}_{B}$ transforms to a total derivative plus terms that are zero on the constrained surface defined by the CF type restrictions (8). Precisely, similar is the situation with the Lagrangian density $\mathcal{L}_{\bar{B}}$ under the nilpotent BRST transformations $s_{b}$.

\section{Comments on CF type restrictions}

It is well-known that a gauge theory is always endowed with a local gauge symmetry that is generated by the first-class constraints in the language of Dirac's prescription for the classification scheme. Thus, the decisive feature of a gauge theory is the existence of first-class constraints on the theory. When any arbitrary $p$-form gauge theory is discussed, within the framework of the BRST formalism, the above local gauge symmetry is traded with the supersymmetric-type (anti-)BRST symmetries $s_{(a) b}$ which turn out to be nilpotent $\left(s_{(a) b}^{2}=0\right)$ of order two. Furthermore, the other sacrosanct feature of the latter symmetries is the absolute anticommutativity (i.e. $s_{b} s_{a b}+s_{a b} s_{b}=$ $0)$. The anticommutativity property is achieved only due to the presence of $\mathrm{CF}$ type restrictions. Thus, the clinching feature of any arbitrary $p$-form gauge theory, within the framework of BRST formalism, is the existence of the (anti-)BRST invariant CF type restrictions. For the Abelian 1-form gauge theory, the CF type restriction is trivial. However, it is non-trivial for all the rest of the gauge theories. Finally, the first-class constraints of the original gauge theory are encoded in the physicality criteria $Q_{b} \mid$ phys $>=0$ where $Q_{b}$ is the conserved and nilpotent BRST charge. This condition, in BRST formalism, enforces all the physical quantum states to be annihilated by the operator form of the first-class constraints of the original theory. 


\section{Conclusions}

In this presentation, it has been emphasized that the Bonora-Tonin's superfield approach $[5,6]$ to BRST formalism always leads to the derivation of the proper (i.e. off-shell nilpotent and absolutely anticommuting) (anti-)BRST symmetry transformations for a given $p$-form gauge theory in any arbitrary $D$-dimensions of spacetime. Furthermore, this geometrical superfield formalism $[5,6]$ necessarily entails upon any arbitrary $D$-dimensional $p$-form gauge theory to be endowed with the (anti-)BRST invariant CF type restriction(s) which, ultimately, lead to the absolute anticommutativity of the (anti-)BRST symmetry transformations and the derivation of the coupled (but equivalent) Lagrangian densities. It turns out that the $\mathrm{CF}$ condition, for the simple case of Abelian $U(1)$ 1-form gauge theory, is trivial. As a consequence, there is a single Lagrangian density for this theory that respects the (anti-)BRST symmetries together. This is not the case, however, for even the non-Abelian $S U(N)$ 1-form gauge theory and all the rest of the (non-)Abelian higher $p$ form $(p \geq 2)$ gauge theories in any arbitrary $D$-dimensions of sapcetime.

\section{Acknowledgement}

Travel support from DST, Govt. of India, is gratefully acknowledged.

\section{References}

[1] Green M. B., Schwarz J. H. and Witten E., Superstring Theory (Cambridge Univ. Press, Cambridge, 1987).

[2] Polchinski J. String Theory (Cambridge Univ. Press, Cambridge, 1998).

[3] Ogievetsky V. I. and Polubarinov I. V., Sov. J. Nucl. Phys. (Yad. Fiz.) 4 (1967) 156.

[4] Ogievetsky V. I. and Polubarinov I. V., Yad. Fiz. 4 (1966) 216.

[5] Bonora L. and Tonin M., Phys. Lett. B 98 (1981) 48.

[6] Bonora L., Pasti P. and Tonin M., Nuovo Cimento A 63 (1981) 353.

[7] Curci G. and Ferrari R., Phys. Lett. B 63 (1976) 91.

[8] Malik R. P., Eur. Phys. J. C 60 (2009) 457, hep-th/0702039.

[9] Bonora L. and Malik R. P., J. Phys.: Math. Theor. 43 (2010) 375403. 